\title{
Hospitalization Costs for Patients Undergoing Orthopedic Surgery Treated With Intravenous Acetaminophen (IV-APAP) Plus Other IV Analgesics or IV Opioid Monotherapy for Postoperative Pain
}

Brett A. Maiese · An T. Pham • Manasee V. Shah • Michael T. Eaddy •

Orsolya E. Lunacsek · George J. Wan

Received: September 19, 2016 / Published online: December 10, 2016

(C) The Author(s) 2016. This article is published with open access at Springerlink.com

\section{ABSTRACT}

Introduction: To assess the impact on hospitalization costs of multimodal analgesia (MMA), including intravenous acetaminophen (IV-APAP), versus IV opioid monotherapy for postoperative pain management in patients undergoing orthopedic surgery.

Methods: Utilizing the Truven Health MarketScan ${ }^{\circledR}$ Hospital Drug Database (HDD), patients undergoing total knee arthroplasty (TKA), total hip arthroplasty (THA), or surgical repair of hip fracture between 1/1/2011 and $8 / 31 / 2014$ were separated into postoperative pain management groups: MMA with IV-APAP

Enhanced content To view enhanced content for this article go to http://www.medengine.com/Redeem/ F427F0601472554F.

B. A. Maiese - M. V. Shah · M. T. Eaddy .

O. E. Lunacsek

Xcenda LLC, Palm Harbor, FL, USA

A. T. Pham $(\bowtie) \cdot$ G. J. Wan

Health Economics and Outcomes Research

Department, Mallinckrodt Pharmaceuticals,

Hampton, NJ, USA

e-mail: an.pham@mallinckrodt.com

Present Address:

M. V. Shah

Mapi Group, Ann Arbor, MI, USA plus other IV analgesics (IV-APAP group) or an IV opioid monotherapy group. All patients could have received oral analgesics. Baseline characteristics and total hospitalization costs were compared. Additionally, an inverse probability treatment weighting [IPTW] with propensity scores analysis further assessed hospitalization cost differences.

Results: The IV-APAP group $(n=33,954)$ and IV opioid monotherapy group $(n=110,300)$ differed significantly $(P<0.0001)$ across baseline characteristics, though the differences may not have been clinically meaningful. Total hospitalization costs (mean \pm standard deviation) were significantly lower for the IV-APAP group than the IV opioid monotherapy group (US\$12,540 $\$ \$ 9564$ vs. $\$ 13,242 \pm \$ 35,825 ; P<0.0001)$. Medical costs accounted for $\$ 701$ of the $\$ 702$ between-group difference. Pharmacy costs were similar between groups. Results of the IPTW-adjusted analysis further supported the statistically significant cost difference.

Conclusions: Patients undergoing orthopedic surgery who received MMA for postoperative pain management, including IV-APAP, had significantly lower total costs than patients 
who received IV opioid monotherapy. This difference was driven by medical costs; importantly, there was no difference in pharmacy costs. Generalizability of the results may be limited to patients admitted to hospitals similar to those included in HDD. Dosing could not be determined, so it was not possible to quantify utilization of IV-APAP or ascertain differences in opioid consumption between the 2 groups. This study did not account for healthcare utilization post-discharge.

Keywords: Hospitalization costs; Intravenous acetaminophen; IV opioid monotherapy; Multimodal analgesia; Orthopedic surgery; Postoperative analgesia; Triple aim

\section{INTRODUCTION}

The Institute for Healthcare Improvement (IHI) Triple Aim requires simultaneous pursuit of (1) improving the patient care experience, (2) advancing population health, and (3) reducing per capita costs of healthcare $[1,2]$. In the context of postoperative pain management, current practice guidelines and recommendations from professional groups and agencies across disciplines such as the American Academy of Orthopaedic Surgeons [3, 4], American Society of Anesthesiologists [5], American College of Critical Care Medicine [6], American Pain Society [7], American Society for Pain Management Nursing [8], Joint Commission [9, 10], and Agency for Healthcare Research and Quality [11] support the use of multimodal analgesia (MMA), which is defined as the use of multiple analgesic medications and nondrug interventions targeting different peripheral or central nervous system sites in the pain pathway [7]. This approach follows the rationale that use of multiple medications may provide additive, or possibly synergistic, analgesic effects compared with a single agent [7]. The goal of MMA is to improve pain control and, by reducing dose levels of any 1 medication, ameliorate side effects [12]. Clinical trials have demonstrated superior pain control and reduced consumption of opioids, as administered via patient-controlled analgesia, with MMA compared with single-agent pain therapy $[13,14]$. Despite the increased intensity of pain management intervention associated with MMA, it may reduce medical costs by improving postoperative clinical outcomes and shortening length of stay (LOS) [15].

In addition to other widely used pain medications, including opioids, nonsteroidal anti-inflammatory drugs (NSAIDs) (used as early as the 1960s [16]) and some neurologic agents (e.g., gabapentin and pregabalin), acetaminophen (APAP), a centrally-acting analgesic agent, is commonly employed in postoperative MMA [7, 17]. In certain patients, including those for whom use of NSAIDs may be inadvisable or contraindicated (e.g., patients with certain cardiac diseases or who have undergone coronary artery bypass graft surgery) [6, 18-20], APAP has been used postoperatively. Oral APAP has been available since 1951 [21]. However, the oral formulation does not meet the needs of all postoperative patients, including those who cannot take oral drugs because of nausea, vomiting, or slow recovery of gastrointestinal function and those whose exposure to opioids or anesthesia during surgery may have impaired drug absorption sufficiently to preclude effective analgesia by the oral route. The intravenous formulation of APAP (IV-APAP) was first approved in the United States (US) in 2010 for the management of mild to moderate pain, 
management of moderate to severe pain with adjunctive opioid analgesics, and reduction of fever and is currently available as a branded product [21]. Besides enabling APAP analgesia in patients who are unable to take the medication orally, IV-APAP offers more efficient and reliable delivery of therapeutic doses than do the oral and rectal forms $[17,22,23]$. Compared with either oral or rectal administration at similar dose levels, IV-APAP yields quicker and higher peak plasma and cerebrospinal drug concentrations [17].

In randomized clinical trials conducted in the US, IV-APAP use in surgical patients has demonstrated analgesic efficacy as evaluated through assessments of pain and pain relief and measurements of opioid consumption, with a favorable safety and tolerability profile [24-27]. More recently, IV-APAP's efficacy, opioid-sparing benefits, and safety in postoperative pain management were confirmed in a Cochrane Collaboration systematic review comprising 75 studies conducted in Africa, Australia, Asia, the US, and Europe [28]. Randomized clinical trials of IV-APAP have also yielded evidence of improved patient satisfaction [29].

The orthopedic surgical procedures total knee arthroplasty (TKA), total hip arthroplasty (THA), and hip repair rank among the 12 most commonly performed inpatient surgical procedures in the US, together accounting for more than 1.4 million hospital stays annually, according to the most recent data (2012) from the Healthcare Cost and Utilization Project [30]. Consequently, the costs, or cost savings, associated with treatment interventions in this orthopedic surgery subpopulation can have important economic implications for healthcare institutions. To assess the hospitalization cost impact of MMA including IV-APAP, an exploratory analysis of patients in a US hospital database who underwent TKA, THA, or hip repair was conducted to compare costs in patients receiving IV-APAP and other IV analgesics as part of MMA, with those receiving IV opioid monotherapy.

\section{METHODS}

\section{Study Design}

This was a retrospective observational analysis of patients who underwent inpatient elective orthopedic surgery at US hospitals that contribute billing data to a national hospital database. This article is based on previously collected data and does not involve any new data collection of human or animal subjects performed by any of the authors.

\section{Data Source}

Data were obtained from the MarketScan ${ }^{\circledR}$ Hospital Drug Database (HDD) (Truven Health Analytics, Ann Arbor, MI, USA). The HDD contains data from the billing systems of approximately 600 participating hospitals, which represent approximately $11 \%$ of all US hospitals [31]. Each record represents an inpatient admission and discharge and includes detailed information on patient demographics, provider specialty, diagnoses, procedures, drug administration, and facility charges. All services recorded by the hospital and all admissions, regardless of the patient's insurance status, are captured. Data are updated monthly with the majority of hospitals reporting discharges at a 45-day lag after the close of the month. 


\section{Study Population}

Patients 18 years of age or older who underwent elective TKA, THA, or surgical repair of hip fracture, as indicated by the principal International Classification of Diseases, 9th Revision, Clinical Modification (ICD-9-CM) procedure code or the Current Procedural Terminology (CPT) code from the index hospitalization (see "Appendix", Table 3 for codes), were identified. Patients were required to have discharge disposition coded as "home" or "other eligible facility" (e.g., other self-care facility, short-term care facility, or home health services). Patients who had evidence of substance abuse disorder (see "Appendix", Table 3) or who used methadone or buprenorphine during the index hospitalization were excluded. Also excluded were patients who underwent more than 1 surgery of interest during the same hospitalization.

\section{Study Cohorts}

Patients were categorized by the postoperative pain management regimen received: a combination of IV-APAP plus other analgesics (which could include both IV and oral analgesics) (IV-APAP group) or IV opioid monotherapy (IV opioid monotherapy group). Patients who also received IV NSAIDs were excluded from the IV opioid group, as this group was intended to represent patients receiving monotherapy with IV opioids. Patients in either cohort could have also received oral analgesics (e.g., hydromorphone, oxycodone, hydrocodone, NSAIDs) as part of the postoperative pain management regimen. To be eligible, patients had to have started IV analgesia on postoperative day 0 (i.e., the same day as surgery).

\section{Variables}

The following data from the HDD were included in this analysis: patient demographics, geographic region, payer type, hospital size, setting type, teaching status, and year of surgical procedure. Cost data included total hospitalization costs, which comprised medical costs and pharmacy costs. Medical costs included medical/surgical supplies, laboratory, imaging, and other costs (including room and board, operating room, therapy/ respiratory therapy, and miscellaneous/routine costs). Costs were analyzed for the index admission only and were adjusted to 2014 US dollars using the medical component of the Consumer Price Index (United States Department of Labor). Costs represent what it costs the hospital to provide care to the patient, independent of insurance type or status.

\section{Statistical Analysis}

Descriptive statistics were used to characterize the study sample during the baseline period and to compare treatment groups in terms of demographic, payer, and hospital characteristics. Differences in categorical variables were assessed using the Chi square test, while differences in continuous variables (including unadjusted differences in hospitalization costs) were assessed using the Student's $t$ test. The a priori statistical significance level was set at $\alpha=0.05$.

In the adjusted analysis of hospitalization costs, inverse probability treatment weighting (IPTW) with propensity scores was used to account for potential confounding while retaining the entire sample of patients. The propensity score was defined as the probability of being treated with IV-APAP, conditional on the individual's baseline characteristics (age at 
index admission, year of index admission, and index payer), and the score was derived from a logistic regression model. A weight was then calculated for each individual as the inverse of the individual's propensity score [32]. These weights were used to create a synthetic sample in which the distribution of measured baseline covariates was independent of treatment assignment. This adjusted analysis controlled for age, gender, region, payer, year of index admission, setting type, teaching status, and hospital size.

\section{RESULTS}

\section{Sample Selection}

Figure 1 summarizes the selection of eligible patients from the entire population of patients who underwent the selected elective orthopedic procedures during the enrollment period and who received IV-APAP as part of an MMA regimen or who received IV opioid monotherapy. In the IV-APAP group, the most common other IV analgesics received were fentanyl, hydromorphone, and morphine while the most common oral analgesics received were celecoxib, hydrocodone, and tramadol (see "Appendix", Tables 4 and 5). The final sample comprised 33,954 patients who received IV-APAP as part of MMA and 110,300 who received IV opioid monotherapy.

\section{Patient Demographic, Payer, and Hospital Characteristics}

Patient demographic, payer, and hospital characteristics are shown in Table 1. Statistically significant differences between treatment groups were noted for all baseline characteristics. However, the large sample sizes confer greater likelihood of attaining statistically significant differences, irrespective of whether numerical differences observed are meaningful in clinical or practical terms [33]. Standardized differences for these baseline characteristics are also included in Table 1. A standardized difference of $10 \%$ (or 0.1 ) is considered equivalent to a $P$ value of 0.05 , indicating insignificant correlation [34]. The only readily discerned noteworthy difference between the treatment groups was that patients in the IV-APAP group tended to have had admissions during the latter 2 years of the study period; in this group, $65.1 \%$ of index admissions occurred in 2013 or 2014 , compared with $33.2 \%$ in the IV opioid monotherapy group.

Unadjusted total, medical, and pharmacy costs for the IV-APAP and IV opioid monotherapy groups are shown in Table 2. Total mean [ \pm standard deviation (SD)] hospitalization cost was significantly lower $(P<0.0001)$ for patients in the IV-APAP group compared with those in the IV opioid monotherapy group $(\$ 12,540 \pm \$ 9564$ vs. $\$ 13,242 \pm \$ 35,825)$, which represents an approximately $\$ 702$ lower total cost for the IV-APAP group. The difference in total costs was almost completely accounted for by medical costs, which were $\$ 701$ lower in the IV-APAP group than in the IV opioid monotherapy group $(P<0.0001)$. Within the medical cost category, the largest between-group absolute difference was in "other" costs (room and board, operating room costs, therapy and respiratory therapy costs, and miscellaneous/routine costs), which were $\$ 571$ lower for the IV-APAP group $(P<0.0001)$. Of note, pharmacy costs were comparable between the treatment groups ( $\$ 486$ for the IV-APAP group and $\$ 488$ for the IV opioid monotherapy group).

In the adjusted analysis using IPTW with propensity scores, total hospitalization costs (Fig. 2) were consistent with the unadjusted analysis, with the IV-APAP group having $\$ 830$ lower total costs than the IV opioid monotherapy group $(P<0.0001)$. 


\section{APAP}

Patients with surgery between January 1, 2011 and August 31, 2014 (index date) $\mathrm{n}=58,296$

Non-elective surgery $n=14,359(24.6 \%)$

Discharge disposition other than home or a facility of interest $n=12,668(21.7 \%)$

Age $<18$ years at admission $\mathrm{n}=392(0.7 \%)$

Substance abuse $\mathrm{n}=646(1.1 \%)$

$>1$ surgery type of interest during the index hospitalization $\mathrm{n}=27(0.05 \%)$

IV-APAP not initiated on postoperative Day 0

$n=3,622(6.2 \%)$

Final Sample (IV-APAP Group) $n=33,954(58.2 \%)$

\section{Opioid Monotherapy Group}

Patients with surgery between January 1, 2011 and August 31, 2014 (index date) $n=345,484$

Non-elective surgery

$\mathrm{n}=119,122(34.5 \%)$

Discharge disposition other than home or a facility of interest

$n=77,246(22.4 \%)$

Age $<18$ years at admission $n=1,761(0.5 \%)$

Substance abuse $n=3,220(0.9 \%)$

$>1$ surgery type of interest during the index hospitalization $\mathrm{n}=97(0.03 \%)$

IV opioid not initiated on postoperative Day 0

$n=7,742(2.2 \%)$

\section{NSAID use}

$n=121,759(35.2 \%)$

Final Sample (IV Opioid Monotherapy Group)

$n=110,300(31.9 \%)$

Some patients were excluded based on multiple criteria

Patients in the IV-APAP group received a combination of IV-APAP plus other analgesics

Substance abuse was defined as having evidence of substance abuse disorder (ICD-9-CM 304.xx, 305.90) during the pre-index/index hospitalization or use of methadone or buprenorphine in addition to other opioids during index hospitalization

Fig. 1 Study sample selection and exclusion criteria. $I V$ intravenous, $I V-A P A P$ intravenous acetaminophen, NSAID nonsteroidal anti-inflammatory drug

\section{DISCUSSION}

In this retrospective observational analysis of more than 140,000 hospital admissions for TKA, THA, or hip fracture repair, costs for patients receiving postoperative MMA with a combination of IV-APAP plus other IV analgesics were compared with costs for patients who received IV opioid monotherapy starting on the day of surgery. Total mean hospitalization costs were significantly lower, by $\$ 702$, for the IV-APAP group than for the IV 
Table 1 Patient demographic, payer, and hospital characteristics for the IV-APAP group and IV opioid monotherapy group

\begin{tabular}{|c|c|c|c|c|c|c|}
\hline Baseline variable & $\begin{array}{l}\text { IV-APAP } \\
(n=33,954)\end{array}$ & & $\begin{array}{l}\text { IV opioid monotherapy } \\
(n=110,300)\end{array}$ & & $P$ value ${ }^{b}$ & $\begin{array}{l}\text { Standardized } \\
\text { difference }^{c}\end{array}$ \\
\hline Age in years (mean, SD) & 62.1 & 12.1 & 61.4 & 12.5 & $<0.0001$ & 0.0569 \\
\hline Female $(n, \%)$ & 19,151 & 56.4 & 60,787 & 55.1 & $<0.0001$ & 0.0260 \\
\hline Year of index admission $(n, \%)$ & & & & & $<0.0001$ & \\
\hline 2011 & 2420 & 7.1 & 40,729 & 36.9 & & -0.7705 \\
\hline 2012 & 9427 & 27.8 & 32,973 & 29.9 & & -0.0470 \\
\hline 2013 & 13,335 & 39.3 & 24,252 & 22.0 & & 0.3818 \\
\hline 2014 & 8772 & 25.8 & 12,346 & 11.2 & & 0.3838 \\
\hline Payer $(n, \%)$ & & & & & $<0.0001$ & \\
\hline Commercial/private & 8234 & 24.3 & 29,898 & 27.1 & & -0.0654 \\
\hline Medicaid & 1914 & 5.6 & 5189 & 4.7 & & 0.0421 \\
\hline Medicare & 15,300 & 45.1 & 49,252 & 44.7 & & 0.0082 \\
\hline Self-pay & 423 & 1.2 & 1227 & 1.1 & & 0.0124 \\
\hline Other & 8083 & 23.8 & 24,734 & 22.4 & & 0.0328 \\
\hline Geographic region $(n, \%)$ & & & & & $<0.0001$ & \\
\hline Midwest & 5772 & 17.0 & 21,032 & 19.1 & & -0.0538 \\
\hline East & 713 & 2.1 & 9089 & 8.2 & & -0.2800 \\
\hline South & 20,802 & 61.3 & 64,917 & 58.9 & & 0.0492 \\
\hline West & 6667 & 19.6 & 15,262 & 13.8 & & 0.1558 \\
\hline Hospital size, beds $(n, \%)$ & & & & & $<0.0001$ & \\
\hline $1-199$ & 8796 & 25.9 & 19,751 & 17.9 & & 0.1943 \\
\hline $200-299$ & 5106 & 15.0 & 17,287 & 15.7 & & -0.0176 \\
\hline $300-499$ & 11,119 & 32.7 & 39,402 & 35.7 & & -0.0627 \\
\hline $500+$ & 8933 & 26.3 & 33,860 & 30.7 & & -0.0973 \\
\hline Hospital type $(n, \%)$ & & & & & $<0.0001$ & \\
\hline Rural & 2235 & 6.6 & 12,883 & 11.7 & & -0.1777 \\
\hline Urban & 31,719 & 93.4 & 97,417 & 88.3 & & 0.1777 \\
\hline Hospital teaching status $(n, \%)$ & & & & & $<0.0001$ & \\
\hline Non-teaching & 28,329 & 83.4 & 92,206 & 83.6 & & -0.0044 \\
\hline Teaching & 5625 & 16.6 & 18,094 & 16.4 & & 0.0044 \\
\hline
\end{tabular}

$A P A P$ acetaminophen, $I V$ intravenous, $S D$ standard deviation

a Patients in the IV-APAP group received a combination of IV-APAP plus other analgesics

b $P$ value for comparison based on a $t$ test for continuous variables and a Chi square test for categorical variables

c Standardized difference equals the difference in means/proportions divided by standard error, with an imbalance defined as absolute value $>0.10$

opioid monotherapy group. This cost difference was driven almost exclusively by medical costs, particularly "other" medical costs, which included room and board, operating room, therapy/respiratory therapy, and miscellaneous/routine costs. Pharmacy costs for the 2 groups were comparable. The treatment groups differed at baseline; however, an adjusted analysis using IPTW weighting with propensity scores confirmed the findings in 
Table 2 Total hospitalization, medical, and pharmacy costs, IV-APAP group and IV opioid monotherapy group

\begin{tabular}{|c|c|c|c|c|c|}
\hline \multirow{2}{*}{$\begin{array}{l}\text { Hospitalization costs, US\$ (mean, SD) } \\
\text { Total }\end{array}$} & \multicolumn{2}{|c|}{$\begin{array}{l}\begin{array}{l}\text { IV-APAP } \\
(n=33,954)\end{array} \\
(n=3\end{array}$} & \multicolumn{2}{|c|}{$\begin{array}{l}\text { IV opioid monotherapy } \\
(n=110,300)\end{array}$} & \multirow{2}{*}{$\begin{array}{l}\text { P value }^{\mathbf{b}} \\
<0.0001\end{array}$} \\
\hline & $\$ 12,540$ & $\$ 9564$ & $\$ 13,242$ & $\$ 35,825$ & \\
\hline Medical $^{\mathrm{c}}$ & $\$ 12,053$ & $\$ 9377$ & $\$ 12,754$ & $\$ 34,870$ & $<0.0001$ \\
\hline Medical/surgical supplies & $\$ 2795$ & $\$ 1870$ & $\$ 2889$ & $\$ 5717$ & $<0.0001$ \\
\hline Laboratory & $\$ 197$ & $\$ 301$ & $\$ 219$ & $\$ 1019$ & $<0.0001$ \\
\hline Imaging & $\$ 91$ & $\$ 129$ & $\$ 105$ & $\$ 238$ & $<0.0001$ \\
\hline Other $^{\mathrm{d}}$ & $\$ 8970$ & $\$ 7922$ & $\$ 9541$ & $\$ 30,735$ & $<0.0001$ \\
\hline Pharmacy & $\$ 486$ & $\$ 488$ & $\$ 488$ & $\$ 1120$ & 0.6786 \\
\hline
\end{tabular}

$A P A P$ acetaminophen, $I V$ intravenous, $S D$ standard deviation

a Patients in the IV-APAP group received a combination of IV-APAP plus other analgesics

b $P$ value for comparison based on a $t$ test for continuous variables

c Medical costs $=$ medical/surgical supplies costs + lab costs + imaging costs + other costs

$\mathrm{d}$ Other costs include room and board, operating room costs, therapy and respiratory therapy costs, and miscellaneous/ routine costs

hospitalization cost differences seen in the initial, unadjusted analysis.

As the acquisition cost of IV-APAP is higher, the economic implications of its utilization have been of interest to healthcare institutions and systems [35]. In the absence of economic data from prospective studies, retrospective pharmacoeconomic analyses and modeling have been conducted to estimate overall cost impact in surgical patient subpopulations. The current study contributes novel health economic data on the use of IV-APAP as part of postoperative MMA from a large population of surgical patients receiving care at a diverse group of approximately 600 participating hospitals throughout the US. Use of a database derived from hospital billing records enables a comprehensive analysis of inpatient costs for both patients receiving MMA including IV-APAP and those receiving IV opioid monotherapy.

Furthermore, these study results are in agreement with previous research, while providing additional real-world evidence to help inform population health decision makers and healthcare providers about the health economic benefits of IV-APAP as part of MMA in the context of treatment protocols and medical policy development. For example, a recent hospital database analysis of patients who received combination IV-APAP plus IV opioids ( $n=174,805)$ with those who received IV opioid monotherapy $(n=311,090) \quad$ [36] corroborate the current study findings of significantly lower hospitalization costs for the patients who received IV-APAP. Another hospital database analysis of patients who underwent elective major joint arthroplasty found that patients who received IV-APAP had total hospitalization costs that were significantly lower than similar patients who did not receive IV-APAP [37]. While the current analysis did not allow for the elucidation of the factors underlying reduced hospitalization cost in IV-APAP-treated patients, previous analyses have found reduced LOS for such patients [36] and significantly fewer adverse events [37].

A possible cost factor more directly related to use of IV-APAP is opioid consumption [38]. 


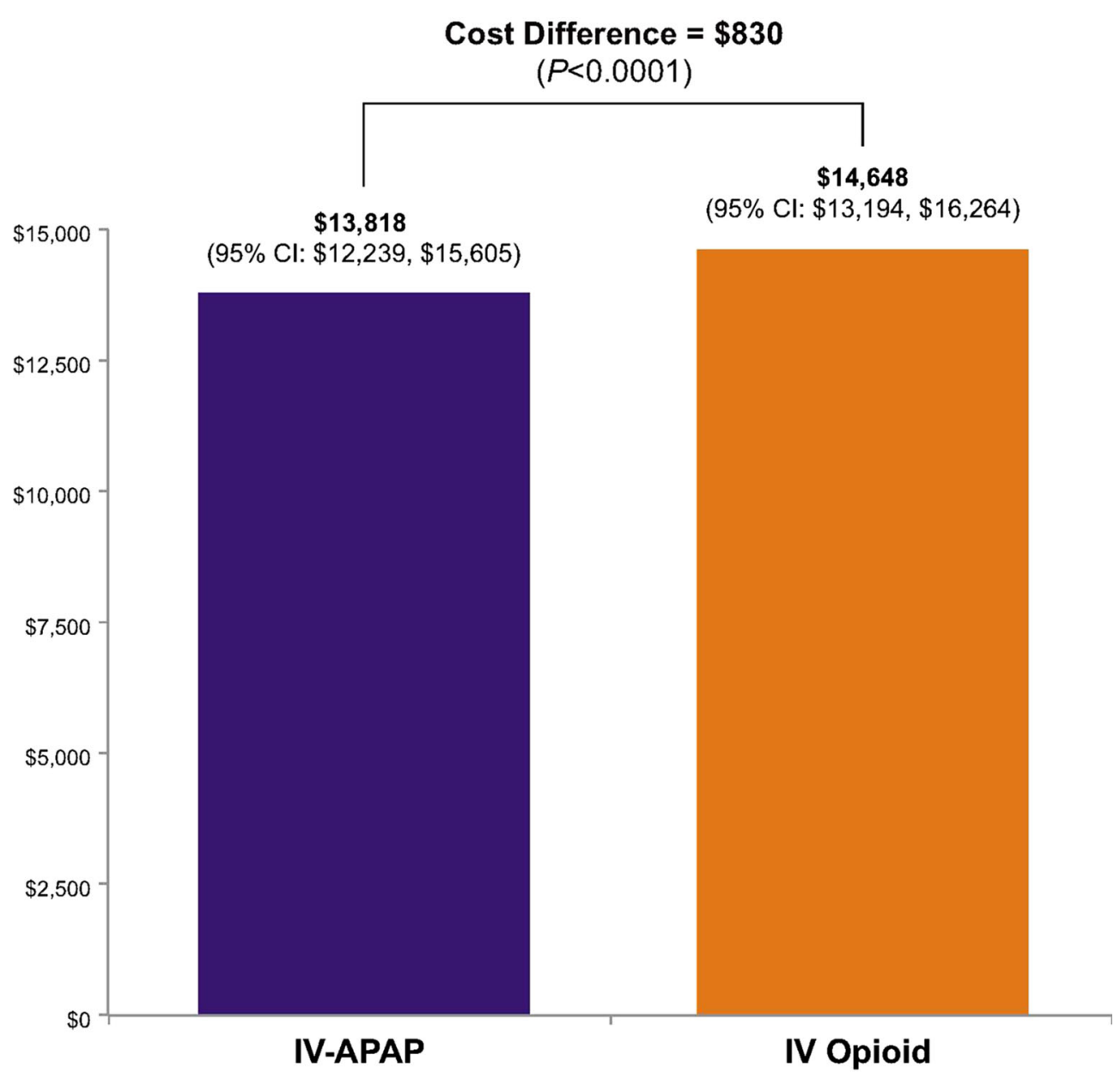

Fig. 2 Total costs for IV-APAP group and IV opioid monotherapy group, based on adjusted analysis using IPTW with propensity scores. $C I$ confidence interval, $I V$ intravenous, $I V$-APAP intravenous acetaminophen, IPTW

Although the average dose or number of doses of IV-APAP or opioids was not evaluated in the presented study, the benefit of IV-APAP in reducing opioid consumption was confirmed in a recent Cochrane Collaboration systematic review and meta-analysis of randomized, double-blind, controlled, single-dose clinical trials of IV-APAP or IV propacetamol for acute postoperative pain in adults or children (75 trials involving 7200 patients) [28]. Patients receiving IV-APAP or IV propacetamol used approximately $26 \%$ fewer opioids (reported as inverse probability treatment weighting. Note: patients in the IV-APAP group received a combination of IV-APAP plus other analgesics. $P$ value was obtained from the IPTW with propensity score analysis output

morphine equivalents) over $4 \mathrm{~h}$ and $16 \%$ fewer opioids over $6 \mathrm{~h}$ than did patients assigned to placebo.

Reduction in morphine requirement with use of IV-APAP formed the basis of a recent pharmacoeconomic modeling study [38]. The study derived data from de-identified records from more than 2 million inpatient surgical encounters at 297 hospitals, including more than 270,000 in which use of IV-APAP was documented. The results indicate that use of IV-APAP and reduction in morphine 
requirement by 1 level (from high to medium, medium to low, or low to none) together reduced rates of opioid-related complications, LOS, and costs for various surgery types. For example, for knee replacement, the calculated reduction in LOS with use of IV-APAP and reduction of morphine requirement by 1 level was 0.77 days, and annual reduction in LOS-associated costs was $\$ 460,000$ from a medium-sized hospital perspective (100-399 beds). Additional cost savings accrued from a nearly $29 \%$ decrease in opioid-related complications with the use of IV-APAP and reduced opioid requirement.

In this study, patients in the IV-APAP group may also have received other IV analgesics along with IV-APAP as part of postoperative MMA (see "Appendix", Table 4 for details on other IV analgesics received), reflecting real-world clinical practices. The findings of the current study support the health economic benefit of IV-APAP use as a part of an MMA strategy and should not be interpreted as a comparison between IV-APAP monotherapy and IV opioid monotherapy. Of note, patients included in either study cohort could have received oral analgesics (see "Appendix", Table 5 for details on oral analgesics received). Therefore, it was not possible to ascertain differences in hospitalization costs directly due to IV-APAP.

It is of note that a larger proportion of the study patients who received IV opioid monotherapy were observed during the earlier part of the study period (approximately $2 / 3$ of the patients had index admissions during 2011-2012), possibly indicating the support of expanded access to and utilization of enhanced treatment options, including MMA with IV-APAP, during the latter years of the study period (2013-2014).

\section{Limitations}

This analysis was limited to patients admitted to those hospitals providing data to the HDD, so generalizability of the results to all patients undergoing orthopedic surgery may be limited. The HDD represents nearly $11 \%$ of the approximately 5,600 registered hospitals in the US [31]; hospitals in the Northeast are slightly overrepresented, compared with the universe of hospitals in the US, as are hospitals with fewer beds, although institutions in the HDD are generally the same with regard to patient age and gender [31, 39]. Additionally, this study assessed hospitalization costs only and could not account for healthcare utilization or costs post-discharge. Additional research is also needed to characterize mechanisms by which MMA including IV-APAP may influence hospitalization costs and other outcomes.

\section{CONCLUSIONS}

In this retrospective observational analysis of a large nationwide hospital database, patients undergoing TKA, THA, or hip fracture repair who received IV-APAP as part of postoperative MMA had significantly lower total hospitalization costs than did patients who received IV opioid monotherapy. This cost difference was driven almost exclusively by significantly lower medical costs; importantly, pharmacy costs for the 2 groups were comparable.

These results suggest that including IV-APAP in MMA for postoperative pain management for these common orthopedic surgical procedures may yield overall cost savings. From a clinician's perspective, the inclusion of IV-APAP as a part of MMA's treatment armamentarium has the potential to assist in 
enhancing recovery and delivering more cost-effective care than IV opioid monotherapy. From a hospital administrator or population health decision maker perspective, MMA may also help reach the goal of the IHI Triple Aim and support current practice guidelines or recommendations from healthcare agencies.

\section{ACKNOWLEDGEMENTS}

Xcenda, LLC, received funding from Mallinckrodt Pharmaceuticals for the conduct of this study and the preparation of the manuscript. Mallinckrodt Pharmaceuticals provided funding for the article processing charges and open access fee. All authors had full access to all of the data in this study and take complete responsibility for the integrity of the data and accuracy of the data analysis. These data were presented in part as an oral e-Poster presentation at the Annual Congress of Enhanced Recovery and Perioperative Medicine, held on April 20-22, 2016 in Washington, DC. Charles Meyer, CMPP, provided writing support for this paper, per arrangement with Xcenda, LLC and with financial support from Mallinckrodt. All named authors meet the International Committee of Medical Journal Editors (ICMJE) criteria for authorship for this manuscript, take responsibility for the integrity of the work as a whole, and have given final approval to the version to be published.

Disclosures. ATP and GJW are employees of Mallinckrodt Pharmaceuticals. BAM, MTE, and OEL are employees of Xcenda, LLC. MVS was an employee at Xcenda, LLC at the time of study design and data analysis.
Compliance with Ethics Guidelines. This article is based on previously conducted data and does not involve any new data collection of human or animal subjects performed by any of the authors.

Author Contributions Statement. MTE, PharmD, PhD: concept design, study oversight, manuscript writing and final approval. OEL, PhD: concept design, data analysis, manuscript review, and final manuscript approval. BAM, PhD, MHS: concept design, data interpretation, manuscript development/writing and review, final manuscript approval. ATP, PharmD, MBA, FAHA: concept design, data review and interpretation, manuscript review, revision, and approval, corresponding author. MVS, MPH: concept design, study oversight, manuscript writing and final manuscript approval. GJW, PhD, MPH: study oversight, data review and interpretation, manuscript review, revision, and final manuscript approval.

Data Availability. The datasets generated during and/or analyzed during the current study are not publicly available due to use restriction of MarketScan data. Summary data are available from the corresponding author on reasonable request.

Open Access. This article is distributed under the terms of the Creative Commons Attribution-NonCommercial 4.0 International License (http://creativecommons.org/licenses/ by-nc/4.0/), which permits any noncommercial use, distribution, and reproduction in any medium, provided you give appropriate credit to the original author(s) and the source, provide a link to the Creative Commons license, and indicate if changes were made. 


\section{APPENDIX}

See Tables 3, 4, 5 .

Table 3 Elective orthopedic surgery procedures and substance abuse codes included in the study by CPT and ICD-9-CM descriptions and codes

\begin{tabular}{|c|c|c|c|c|}
\hline Description & CPT & & ICD-9-CM & \\
\hline $\begin{array}{l}\text { Total knee } \\
\text { arthroplasty/total knee } \\
\text { replacement }\end{array}$ & $\begin{array}{l}\text { Arthroplasty, knee, condyle, and plateau; } \\
\text { medial AND lateral compartments, } \\
\text { with or without patella resurfacing } \\
\text { (total knee arthroplasty) }\end{array}$ & 27447 & Total knee replacement & 81.54 \\
\hline $\begin{array}{l}\text { Total hip } \\
\text { arthroplasty/total hip } \\
\text { replacement }\end{array}$ & $\begin{array}{l}\text { Arthroplasty, acetabular, and proximal } \\
\text { femoral prosthetic replacement (total } \\
\text { hip arthroplasty), with or without } \\
\text { autograft or allograft }\end{array}$ & 27130 & Total hip replacement & 81.51 \\
\hline \multirow{4}{*}{$\begin{array}{l}\text { Surgical repair of hip } \\
\text { fracture (open } \\
\text { reduction and internal } \\
\text { fixation, etc) }\end{array}$} & $\begin{array}{l}\text { Open treatment of posterior or anterior } \\
\text { acetabular wall fracture, with internal } \\
\text { fixation }\end{array}$ & 27226 & $\begin{array}{l}\text { Open reduction of fracture of } \\
\text { femur without internal fixation }\end{array}$ & 79.25 \\
\hline & $\begin{array}{l}\text { Open treatment of acetabular } \\
\text { fracture(s) involving anterior or } \\
\text { posterior (1) column, or a fracture } \\
\text { running transversely across the } \\
\text { acetabulum, with internal fixation }\end{array}$ & 27227 & $\begin{array}{l}\text { Open reduction of fracture of } \\
\text { femur with internal fixation }\end{array}$ & 79.35 \\
\hline & - & - & $\begin{array}{l}\text { Open reduction of fracture of } \\
\text { other specified bone, except } \\
\text { facial bones, without internal } \\
\text { fixation }\end{array}$ & 79.29 \\
\hline & - & - & $\begin{array}{l}\text { Open reduction of fracture of } \\
\text { other specified bone, except } \\
\text { facial bones, with internal } \\
\text { fixation }\end{array}$ & 79.39 \\
\hline Substance abuse disorder & - & - & Drug dependence & $304 . x x$ \\
\hline Substance abuse disorder & - & - & $\begin{array}{l}\text { Nondependent other mixed or } \\
\text { unspecified drug abuse, other, } \\
\text { mixed, or unspecified drug } \\
\text { abuse }\end{array}$ & 305.90 \\
\hline
\end{tabular}

$C P T$ current procedural terminology, ICD-9-CM international classification of diseases, 9th Revision, Clinical Modification 
Table 4 Other IV analgesics received by the IV-APAP group $(n=33,954)$

\begin{tabular}{lrr}
\hline IV analgesics & \multicolumn{1}{l}{$\boldsymbol{n}$} & \% \\
\hline Fentanyl & 26,439 & 77.9 \\
Hydromorphone & 20,683 & 60.9 \\
Morphine & 17,500 & 51.5 \\
Keterolac & 13,334 & 39.3 \\
Meperidine & 2166 & 6.4 \\
Sufentanil & 1248 & 3.7 \\
Remifentanil & 1081 & 3.2 \\
Ibuprofen & 802 & 2.4 \\
Nalbuphine & 170 & 0.5 \\
\hline
\end{tabular}

$A P A P$ acetaminophen, $I V$ intravenous

Table 5 Oral analgesics received by the IV-APAP group and IV opioid monotherapy group

\begin{tabular}{|c|c|c|c|c|}
\hline \multirow[t]{2}{*}{ Oral Analgesics } & \multicolumn{2}{|c|}{$\begin{array}{l}\text { IV-APAP } \\
(n=33,954)\end{array}$} & \multicolumn{2}{|c|}{$\begin{array}{l}\text { IV opioid } \\
\text { monotherapy } \\
(n=110,300)\end{array}$} \\
\hline & $n$ & $\%$ & $\bar{n}$ & $\%$ \\
\hline Celecoxib & 7789 & 22.9 & 16,176 & 14.7 \\
\hline $\begin{array}{l}\text { Hydrocodone bitartrate/ } \\
\text { APAP }\end{array}$ & 4225 & 12.4 & 18.017 & 16.3 \\
\hline Tramadol HCL & 3812 & 11.2 & 2839 & 2.6 \\
\hline Aspirin & 3010 & 8.9 & 4874 & 4.4 \\
\hline Methocarbamol & 1416 & 4.2 & 5145 & 4.7 \\
\hline Hydromorphone HCL & 1154 & 3.4 & 1670 & 1.5 \\
\hline Morphine sulfate & 857 & 2.5 & 1480 & 1.3 \\
\hline $\begin{array}{c}\text { Narcotic analgesic } \\
\text { combinations }\end{array}$ & 424 & 1.2 & 4367 & 4.0 \\
\hline Fentanyl & 395 & 1.2 & 2172 & 2.0 \\
\hline
\end{tabular}

\section{REFERENCES}

1. Berwick DM, Nolan TW, Whittington J. The triple aim: care, health, and cost. Health Aff (Millwood). 2008;27(3):759-69.
2. Institute for Healthcare Improvement (IHI). The IHI Triple Aim. http://www.ihi.org/engage/initiatives/ TripleAim/Pages/default.aspx. 2016. Accessed August 3, 2016.

3. American Academy of Orthopaedic Surgeons. Management of hip fractures in the elderly: Evidence-based clinical practice guideline. http:// www.aaos.org/cc_files/aaosorg/research/guidelines/ hipfxguideline.pdf. 2014. Accessed June 12, 2016.

4. American Academy of Orthopaedic Surgeons. Current strategies in anesthesia and analgesia for total knee arthroplasty. J Am Acad Orthop Surg. 2016;24(2):60-73.

5. American Society of Anesthesiologists Task Force on Acute Pain Management. Practice guidelines for acute pain management in the perioperative setting: an updated report by the American Society of Anesthesiologists Task Force on Acute Pain Management. Anesthesiology. 2012;116(2):248-73.

6. Barr J, Fraser GL, Puntillo K, et al. Clinical practice guidelines for the management of pain, agitation, and delirium in adult patients in the intensive care unit. Crit Care Med. 2013;41:263-306.

7. Chou R, Gordon DB, de Leon-Casasola OA, et al. Management of postoperative pain: a Clinical practice guideline from the American Pain Society, the American Society of Regional Anesthesia and Pain Medicine, and the American Society of Anesthesiologists' Committee on Regional Anesthesia, Executive Committee, and Administrative Council. J Pain. 2016;17(2):131-57.

8. Jarzyna D, Jungquist CR, Pasero C, et al. American Society for Pain Management Nursing guidelines on monitoring for opioid-induced sedation and respiratory depression. Pain Manag Nurs. 2011;12:118-45.

9. Joint Commission. Safe use of opioids in hospitals. Sentinel Event Alert. 2012;49:1-5. http://www. jointcommission.org/assets/1/18/SEA_49_opioids_ 8_2_12_final.pdf. Accessed June 28, 2016.

10. Joint Commission. Clarification of the pain management standard. Joint Commission Perspectives. 2014;34(11):11. http://www. jointcommission.org/assets/1/18/Clarification_of_ the_Pain_Management_Standard.pdf. Accessed June 28, 2016.

11. Hughes RD, ed. Patient Safety and Quality: An Evidence-Based Handbook for Nurses. 2008. Rockville, MD: Agency for Healthcare Research and Quality. 
12. Chandrakantan A, Glass PS. Multimodal therapies for postoperative nausea and vomiting, and pain. $\mathrm{Br}$ J Anaesth. 2011;107(Suppl 1):i27-40.

13. Elia N, Lysakowski C, Tramèr MR. Does multimodal analgesia with acetaminophen, nonsteroidal antiinflammatory drugs, or selective cyclooxygenase-2 inhibitors and patient-controlled analgesia morphine offer advantages over morphine alone? Meta-analyses of randomized trials. Anesthesiology. 2005;103(6):1296-304.

14. Maund E, McDaid C, Rice S, Wright K, Jenkins B, Woolacott N. Paracetamol and selective and non-selective non-steroidal anti-inflammatory drugs for the reduction in morphine-related side-effects after major surgery: a systematic review. Br J Anaesth. 2011;106(3):292-7.

15. Duncan CM, Hall Long K, Warner DO, Hebl JR. The economic implications of a multimodal analgesic regimen for patients undergoing major orthopedic surgery: a comparative study of direct costs. Reg Anesth Pain Med. 2009;34(4):301-7.

16. Buer JK. Origins and impact of the term 'NSAID'. Inflammopharmacology. 2014;22(5):263-7.

17. Singla NK, Parulan C, Samson R, et al. Plasma and cerebrospinal fluid pharmacokinetic parameters after single-dose administration of intravenous, oral, or rectal acetaminophen. Pain Pract. 2012;12(7):523-32.

18. Cattabriga I, Pacini D, Lamazza G, et al. Intravenous paracetamol as adjunctive treatment for postoperative pain after cardiac surgery: a double blind randomized controlled trial. Eur J Cardiothorac Surg. 2007;32(3):527-31.

19. Ketorolac tromethamine injection. USP [prescribing information]. Schaumberg, IL: Sagent Pharmaceuticals; 2015.

20. Caldolor (ibuprofen) injection, for intravenous use [prescribing information]. Nashville, TN: Cumberland Pharmaceuticals Inc; April 2016.

21. Ofirmev injection [prescribing information]. Hazelwood, MO: Mallinckrodt Pharmaceuticals; December 2014.

22. Møller PL, Sindet-Pedersen S, Petersen CT, Juhl GI, Dillenschneider A, Skoglund LA. Onset of acetaminophen analgesia: comparison of oral and intravenous routes after third molar surgery. Br J Anaesth. 2005;94(5):642-8.

23. van der Westhuizen J, Kuo PY, Reed PW, Holder K. Randomised controlled trial comparing oral and intravenous paracetamol (acetaminophen) plasma levels when given as preoperative analgesia. Anaesth Intensive Care. 2011;39:242-6.

24. Sinatra RS, Jahr JS, Reynolds LW, Viscusi ER, Groudine SB, Payen-Champenois C. Efficacy and safety of single and repeated administration of 1 gram intravenous acetaminophen injection (paracetamol) for pain management after major orthopedic surgery. Anesthesiology. 2005;102(4):822-31.

25. Wininger SJ, Miller H, Minkowitz HS, Royal MA, Ang RY, Breitmeyer JB, Singla NK. A randomized, doubleblind, placebo-controlled, multicenter, repeat-dose study of two intravenous acetaminophen dosing regimens for the treatment of pain after abdominal laparoscopic surgery. Clin Ther. 2010;32(14):2348-69.

26. Sinatra RS, Jahr JS, Reynolds L, Groudine SB, Royal MA, Breitmeyer JB, Viscusi ER. Intravenous acetaminophen for pain after major orthopedic surgery: an expanded analysis. Pain Pract. 2012;12(5):357-65.

27. Singla NK, Hale ME, Davis JC, et al. IV acetaminophen: efficacy of a single dose for postoperative pain after hip arthroplasty: subset data analysis of 2 unpublished randomized clinical trials. Am J Ther. 2015;22(1):2-10.

28. McNicol ED, Ferguson MC, Haroutounian S, Carr DB, Schumann R. Single dose intravenous paracetamol or intravenous propacetamol for postoperative pain. Cochrane Database Syst Rev. 2016;23(5):CD007126.

29. Apfel CC, Souza K, Portillo J, Dalal P, Bergese SD. Patient satisfaction with intravenous acetaminophen: a pooled analysis of five randomized, placebo-controlled studies in the acute postoperative setting. J Healthc Qual. 2015;37(3):155-62.

30. Fingar KR, Stocks C, Weiss AJ, Steiner CA. Most frequent operating room procedures performed in U.S. hospitals, 2003-2012. HCUP Statistical Brief \#186. December 2014. Agency for Healthcare Research and Quality, Rockville, MD. https:// www.hcup-us.ahrq.gov/reports/statbriefs/sb186Operating-Room-Procedures-United-States-2012. pdf. Accessed May 21, 2016.

31. American Hospital Association (AHA). Fast facts on US hospitals. http://www.aha.org/research/rc/statstudies/fast-facts.shtml. 2016. Accessed March 9, 2016.

32. Austin PC. An Introduction to propensity score methods for reducing the effects of confounding in observational studies. Multivariate Behav Res. 2011;46(3):399-424. 
33. Lin M, Lucas HC Jr, Shmueli G. Too big to fail: large samples and the p-value problem. Inf Sys Res. 2013;24:906.

34. Austin PC. Using the standardized difference to compare the prevalence of a binary variable between two groups in observational research. Comm Stat Simulat Comput. 2009;38:1228-34.

35. Malesker MA, Bruckner AL, Loggie B, Hilleman DE. Intravenous acetaminophen: Assessment of medication utilization evaluation data in peri-operative pain management. J Surg. 2015;10(4):257-61.

36. Hansen RN, Pham A, Strassels SA, Balaban S, Wan GJ. Comparative analysis of length of stay and inpatient costs for orthopedic surgery patients treated with IV acetaminophen and IV opioids vs. IV opioids alone for post-operative pain. Adv Ther. 2016;. doi:10.1007/s12325-016-0368-8.
37. Apfel C, Jahr JR, Kelly CL, Ang RY, Oderda GM. Effect of I.V. acetaminophen on total hip or knee replacement surgery: a case-matched evaluation of a national patient database. Am J Health Syst Pharm. 2015;72(22):1961-8.

38. Shaffer EE, Pham A, Woldman RL, et al. Estimating the effect of intravenous acetaminophen for postoperative pain management on length of stay and inpatient hospital costs. Adv Ther. 2017;33:2211. doi:10.1007/s12325-016-0438-y.

39. Centers for Disease Control and Prevention. National Hospital Discharge Survey: Data Highlights-Selected Tables. http://www.cdc.gov/ nchs/nhds/nhds_tables.htm. 2010. Accessed March 9, 2016. 